\title{
Generation of 3D Sensitivity Map for a Helical ECT Sensor in Annular Space
}

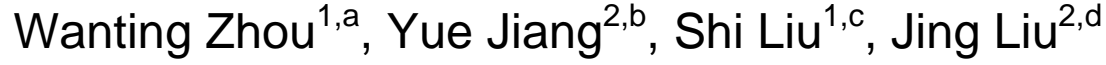 \\ ${ }^{1}$ School of Control and Computer Engineering, North China Electric Power University, Beijing \\ 102206, China; \\ ${ }^{2}$ School of Energy, Power and Mechanical Engineering, North China Electric Power University, \\ Beijing 102206, China; \\ a709675520@qq.com, b769400892@qq.com, cliushidr@yahoo.com, d961974939@qq.com
}

Keywords: Annular space, three-dimensional, ECT, helical sensor, sensitivity map.

\begin{abstract}
Annular space has been applied widely in industrial processes. However, the complexity of thermal process and the variety of influential factors lead to the difficulties. In addition, the sensor installation as well as the weak detection signal due to the barrier increases the detection issues. In order to achieve the visual monitoring of pneumatic transport in annular space, a helical structure sensor based on the direct three-dimensional(3D) Electrical Capacitance Tomography(ECT) is designed in this paper. The 3D sensitivity map in annular space is calculated via the potential value obtained from the finite element analysis software, Ansoft. According to the figures of potential and sensitivity field distribution, the relative characteristic is summarized, which is the foundation to reconstruct the direct 3D image accurately.
\end{abstract}

\section{Introduction}

As an important part of sustainable economic development, pneumatic conveying efficiency could be improved by visualization monitoring ${ }^{[1]}$. Currently, the study focuses on the relatively simple space of round or square. However, the study in annular space is limited, which is widespread as well, such as the horizontal drilling technology in oil exploitation, the air-solid flow process in annular furnace of circulating fluidized bed and so on. Because of the complication of flow field and the variety of influential factors in it lead to the difficulties. In addition, the sensor installation as well as the weak detection signal increases the detection issues.

Electrical Capacitance Tomography(ECT) is one kind of visual monitoring technology. Researchers have made some achievements, especially the sensitivity map calculation. For instance, Shi Liu et al. ${ }^{[2-6]}$ optimized ECT sensor to improve the accuracy of sensitivity map. Yang $\mathrm{Lu}^{[7]}$ analyzed the effects of different conditions on the sensitivity map. However, the two-dimensional ECT technology cannot meet the requirement of detection, and the 3D ECT technology develops gradually, which is divided into indirect and direct imagining. There is only a few articles referring to it, particularly the direct one. For example, Yifan Wang ${ }^{[8]}$ has studied calculation of 3D sensitivity map, optimization of reconstruction algorithm and improvement of the structure of sensor. Zhendong $\operatorname{Lin}^{[9]}$ has mainly studied the effect of sensor's structure on sensitivity map. Z.Ye ${ }^{[10]}$ from Bath University has analyzed the imaging resolution using direct 3D technology. The capacitor electrodes were arranged on a cube's six sides. However, layered arrangement in enclosed space is difficult to adapt industrial environments, and the study of three-dimensional ECT in the annular space is still in the blank stage.

In this paper, a sensor with helical structure is designed to detect in annular space, using the direct 3D ECT. The 3D potential and sensitivity map distribution is generated. The sensitivity map matrix is indispensable in ECT imaging, including both the positive and the inverse problems. The accuracy and precision of that affect image quality directly and the analysis of thermal characteristic further. Therefore, generating of the three-dimensional sensitivity field is significant. 


\section{Theoretical Analysis}

\section{Overview of ECT}

The paper adopts the direct 3D ECT. Its mechanism is that if the shape and distribution of multiphase flow change, measured capacitance value between each pair of electrodes will follow the variation. Therefore, it is feasible to reconstruct the image of multiphase flow distribution via some reconstruction algorithm.

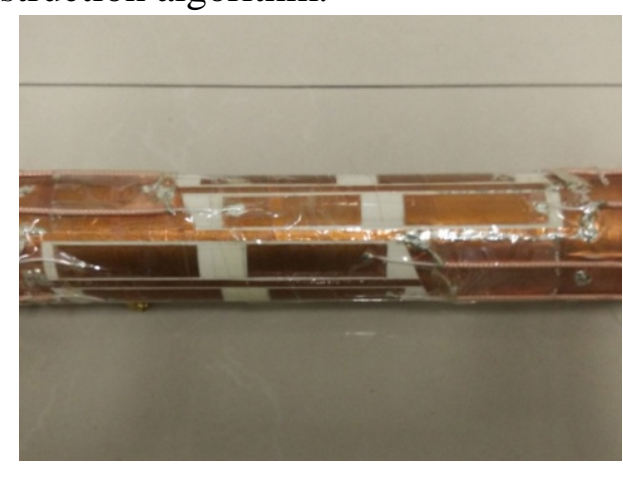

Fig. 1 Structure of sensor

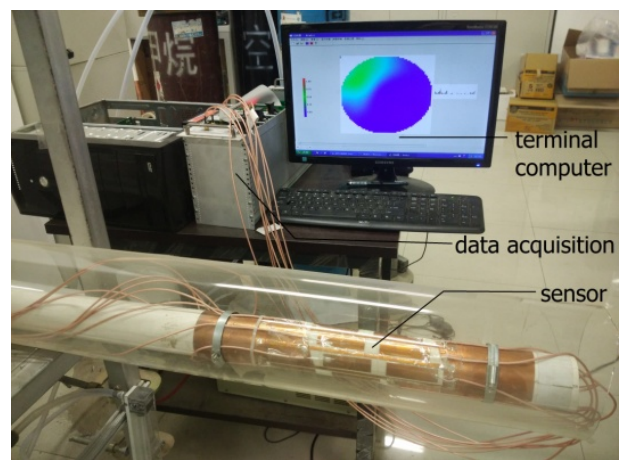

Fig. 2 ECT system

ECT system consists of sensor part, data acquisition and processing part and terminal computer for imaging ${ }^{[11]}$, shown in Fig. 1 and Fig. 2. ECT image reconstruction is the most important part which is divided into positive and inverse problems, and the imaging algorithm could be expressed by the following formula ${ }^{[12]}$.

$$
\mathrm{C}=\mathrm{SG}
$$

where $\mathrm{C}$ is the capacitance value matrix between each pair of electrodes, $\mathrm{S}$ is the sensitivity map, and $\mathrm{G}$ is the dielectric constant matrix. The positive problem is to solve $\mathrm{C}$ from $\mathrm{G}$ and $\mathrm{S}$. Inversely, the inverse problem is to solve $\mathrm{G}$ from $\mathrm{C}$ and $\mathrm{S}$. It can be known that the inverse matrix of $\mathrm{S}$ does not exist, which also causes the difficulty of solving the inverse problem. Therefore, the calculation of the sensitivity map affects the quality of reconstructed image directly.

\section{Direct and Indirect 3D ECT}

In recent years, three-dimensional ECT technology gradually developed, which is mainly divided into two categories ${ }^{[13]}$.

One is the indirect 3D ECT. The approximate 3D image can be obtained from a series of twodimensional images using interpolation, which is reconstructed by measuring the capacitance between the electrodes in the same layer. However, this method has great deviation, particularly the insensitivity in the axial direction.

The other one is the direct one. It requires capacitance between the electrodes both in the same and different layers. In this method, reconstructed image contains the entire 3D space information and the axial resolution is greatly improved. It is sensible to the change at any point, even between the layers. It is adopted in this paper.

\section{Solution of 3D Sensitivity Map}

There are various methods to obtain sensitivity map, such as the capacitance method ${ }^{[14]}$. In this paper, sensitivity map is calculated via potential distribution.

The nature relationship between the changes of medium and capacitance values is represented by the following:

$$
\Delta \mathrm{C}_{\mathrm{ij}}=-\frac{\Delta \varepsilon \int_{\sigma} \Delta \phi_{\mathrm{i}} \cdot \Delta \phi_{\mathrm{j}} \mathrm{ds}}{\mathrm{V}^{2}}+\mathrm{O}\left((\Delta \varepsilon)^{2}\right)
$$

According to the basic principles of ECT:

$$
\Delta \mathrm{C}=\mathrm{S} \Delta \varepsilon
$$

Then, a mathematical model of sensitivity map in region $\sigma$ is obtained:

$$
\mathrm{S}_{\mathrm{ij}}(\sigma)=\frac{\Delta \mathrm{C}_{\mathrm{ij}}}{\Delta \varepsilon} \approx-\frac{\int_{\mathrm{v}} \Delta \phi_{\mathrm{i}} \cdot \Delta \phi_{\mathrm{j}} \mathrm{ds}}{\mathrm{V}^{2}}=\frac{\overline{\mathrm{E}_{1}}(\mathrm{x}, \mathrm{y}, \mathrm{z}) \cdot \overline{\mathrm{E}_{\mathrm{j}}}(\mathrm{x}, \mathrm{y}, \mathrm{z})}{\mathrm{v}_{\mathrm{i}} \cdot \mathrm{v}_{\mathrm{j}}}=\frac{\overline{\mathrm{E}_{1}}(\mathrm{x}, \mathrm{y}, \mathrm{z})}{\mathrm{v}_{\mathrm{i}} \cdot} \cdot \frac{\overline{\mathrm{E}_{\mathrm{j}}}(\mathrm{x}, \mathrm{y}, \mathrm{z})}{\mathrm{v}_{\mathrm{j}}}
$$


Where $\phi_{\mathrm{i}} 、 \phi_{\mathrm{j}}$ denote the potential distribution while voltage $\mathrm{V}$ applied to NO.i or NO.j electrode with the rest is grounded. $\bar{E}_{1}(x, y, z)$ represents the value of electric field intensity at this point. Domain $\sigma$ can be regarded as the pixel of the image.

In this paper, sensitivity map is calculated by the method described above. In Ansoft, a 3D potential gradient distribution which means electric field intensity can be obtained, and then sensitivity map is generated in postprocessing.

\section{Generation of 3D sensitivity map based on Ansoft}

\section{The Structure of Sensor}

In this paper, an ECT sensor with helical structure is designed for detecting in annular space. It consists of internal and external insulation pipes, the metal electrode arrays attached to the inner pipe wall as well as shield. In particular, the metal electrode array consists of 16 electrodes with helical distribution and there are 8 electrodes in each circle. Shield consists of shielding case inside the inner pipe and outside the outer one, trapezoidal shield electrodes arranged on the above and below detection electrodes and radial shield electrodes mounted between the detection electrodes in the axial direction. As shown in Fig. 3, the annular area between two pipes is the measured space.

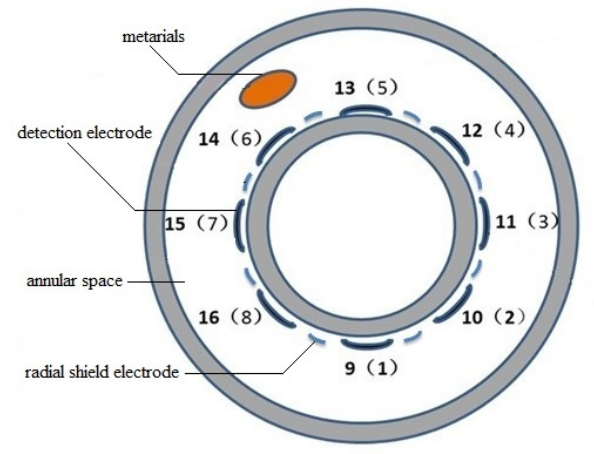

Fig. 3 The top view of the sensor

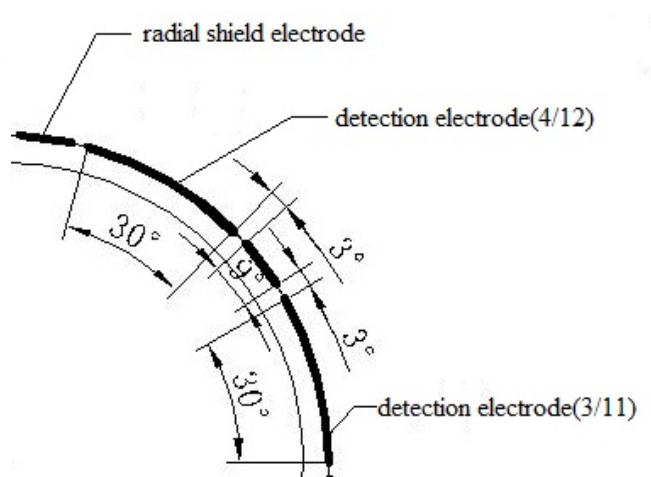

Fig. 4 The size of the sensor

Specific parameters: (1) The diameter of the outer pipe is $150 \mathrm{~mm}$, and the inner one is $63 \mathrm{~mm}$ (2) The detection electrode is 60 millimeters high and 30 degrees angle. The height difference between each two adjacent electrodes is $20 \mathrm{~mm}$. (3) The radial shield electrode is 9 degrees angle. Other dimensions are shown in Fig. 4.

The innovation of the helical structure sensor is that breaking the layered arrangement. The sensor can also get accurate information about section of any direction at any point. Changes of some characteristics of material, such as shape, position, speed and concentration in 3D space, can be reflected on the image, that improves the sensitivity and axial resolution.

Simulation of 3D Sensitivity Map

Step1. Design and build three-dimensional sensor model via Ansoft shown in Fig. 5.

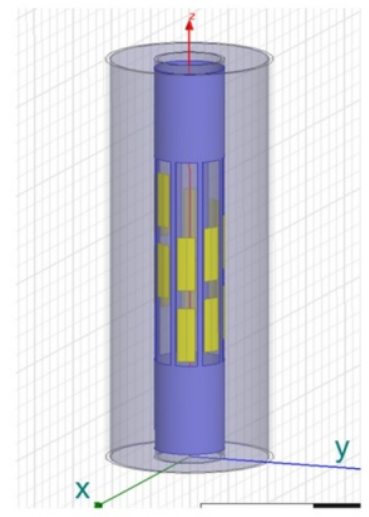

Fig. 5 3D sensor model built by Ansoft 
Step2. Electrostatics solver is set and the materials of outer pipe and inner pipe are glass and PVC respectively. The annular space is set to vacuum.

Step3. The default natural boundary conditions are set. No.1 electrode is taken as the excitation source with $15 \mathrm{~V}$, while the other 15 ones and shield are set to $0 \mathrm{~V}$. Then No.2 is taken as excitation source, and so do for 16 sets.

Step4. Tetrahedral element is adopted for meshing and refined the annular space specially. The final number of grids is about 3 million. Twelve-step calculation and convergence target of $0.1 \%$ is the solution parameters.

\section{Results and discussions}

\section{Potential Distribution in Annular Space}

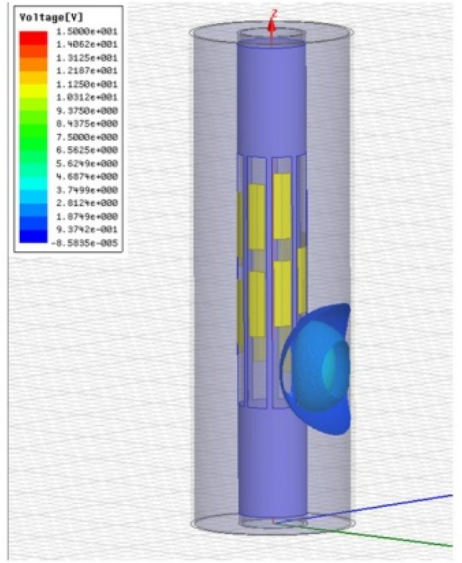

a. side view

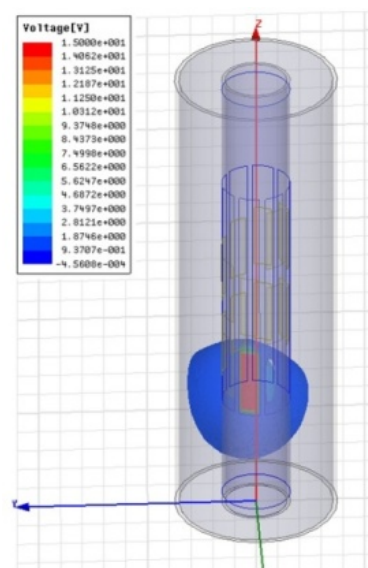

b. back view

Fig. 6 Potential distribution in annular space

The potential distribution in the annular space is shown from different angles in Fig. 6, which is generated from applying voltage on NO.1 electrode. Around NO.1 electrode, three equal potential surfaces can be seen in 3D space, which provides more visual information than power lines in 2D space. Additionally, they presents different colors. The outermost potential value represented by dark blue is relatively low. The closer to the excitation source, the higher potential is. To be specific, blue becomes shallow gradually, until the position of the most close to the electrode presents the red center with green edge. Meanwhile it can be seen that equal potential surfaces become smooth obviously, resulted from the continuity of electric field, which also reflects the soft-field characteristic.

3D Sensitivity Map

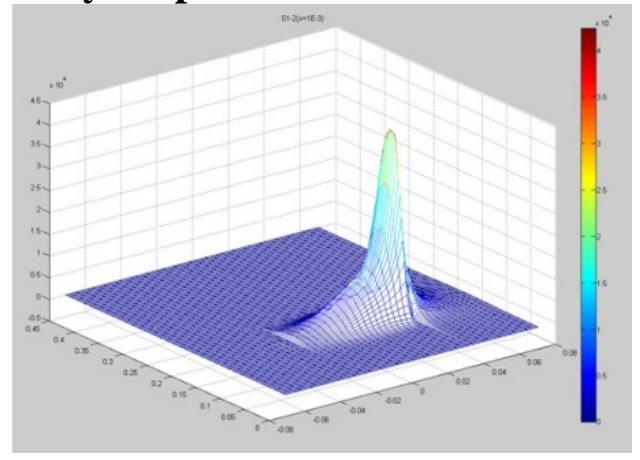

a. Between No.1 and 2 electrodes

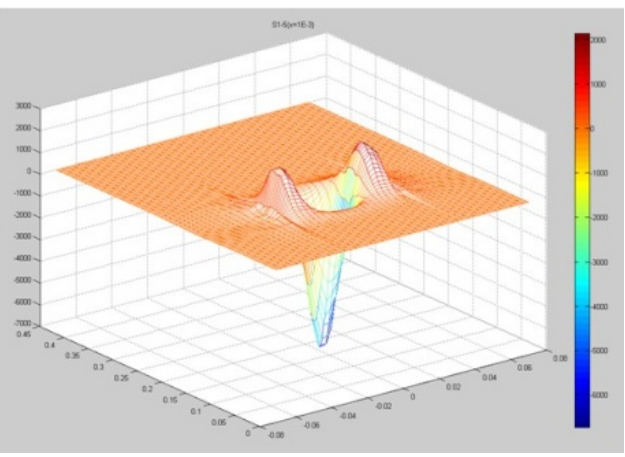

b. Between No.1 and 5 electrodes 


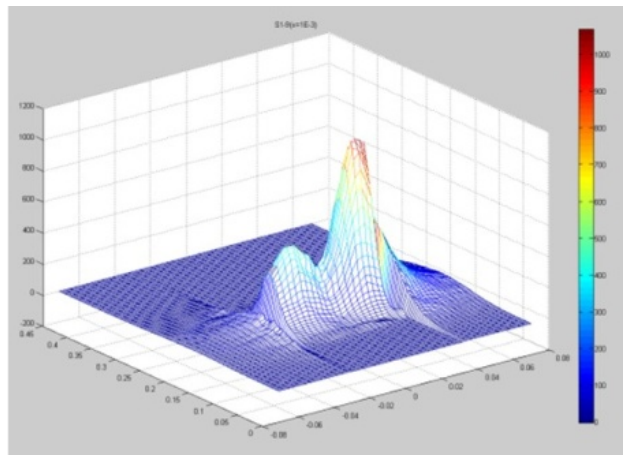

c. Between No.1 and 9 electrodes

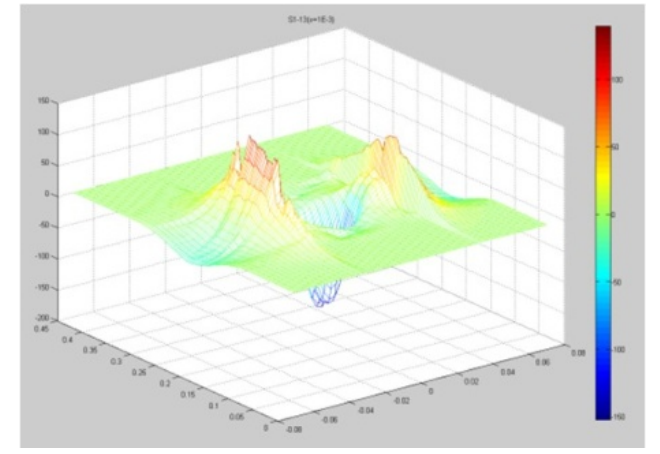

d. Between No.1 and 13 electrodes

Fig. 7 The typical 3D sensitivity map

The direct 3D ECT sensitivity map matrix can be generated by the potential distribution shown in Fig. 6. It is taken to image for analysis in MATLAB that the data of sensitivity map on the $y-z$ surface, shown in Fig. 7.

The general characteristics is as follows. Initially, figures indicate the soft-field characteristic of ECT. That means that the sensitivity map value presents both positive and negative. It is normal that negative values exist and it should not be return to zero. Besides, it shows that the peak of each figure varies hugely, even an order of magnitude. Specifically, the peak of sensitivity map between adjacent electrodes is large and a slight change of dielectric constant will influence the capacitance, which represents higher sensitivity. However, the peak value between opposite electrodes or more separate electrodes is relatively small.

Detailed analysis is as follows. Firstly, the sensitivity map between adjacent electrodes(shown in Fig. 7(a)) has a single peak. The closer to the electrode, the greater the value is. Secondly, that between the opposite ones in the same circle(shown in Fig. 7(b)) has two symmetrical peaks and concave in the middle. It is indicated that high sensitivity area is close to the electrode and sensitivity is decreased as far away from the electrode. Thirdly, that between two electrodes which are at the same point in $x-y$ plane in different circles(shown in Fig. 7(c)) is bimodal. Finally, that between the opposite ones in the different circle is shown in Fig. 7(d). It owns the similar shape to Fig. 7(b), but less smooth than it on account of the noise produced from the height difference between electrodes probably.

Sensitive map generated above is a 3D matrix. Therefore, its section of any direction can be obtained, not limited to parallel to the axis, which is not repeated here.

\section{Conclusions}

A 3D helical structure ECT sensor is proposed in this paper, which can be applied widely to visualization monitoring in complex annular space. The sensitivity map matrix is generated, providing the basis for direct 3D ECT image reconstruction. The analysis of potential distribution and sensitivity map verifies some characteristic.

\section{Acknowledgement}

This paper is finished with the support of the project "Overseas Expertise Introduction Program for Disciplines Innovation in Universities”, and the deepest gratitude of authors goes first and foremost to Professor Shi Liu.

\section{References}

[1] Zhousheng Yu. Pneumatic conveying and application[J]. 1989(in Chinese).

[2] Haigang Wang, Shi Liu, Fan Jiang, et al. 3D imaging with electrical capacitance tomography in cyclone separators[J]. Journal of Engineering Thermophysics, 2006, 27(1):177-179(in Chinese). 
[3] Huaxiang Wang, Lifeng Zhang, Xueming Zhu. Optimum design of array electrode for ECT system[J]. Journal of Tianjin University(Science and Technology), 2003, 36(3):307-310(in Chinese). [4] Hua Yan, Fuqun Shao, Shi Wang. The optimizing design of capacitance tomography sensors[J]. Chinese Journal of Scientific Instrument, 2000, 21(02):139-141(in Chinese).

[5] Fuqun Shao, Yanli Gao, Yonggao Zhang, et al. Analysis and optimization of ECT sensor using orthogonal experiment with interaction of factors[J]. Journal of Northeastern University(Natural Science), 2003, 24(2):103-106(in Chinese).

[6] Hongxing Guo, Shengsheng Yu, Jixian, Feng, et al. Parameter optimizing of multielectrode capacitive transducers[J]. Wuhan University Journal of Natural Sciences, 2004, 46(5):637-640(in Chinese).

[7] Yang Lu. Research on optimization of sensor structure parameters of direct 3D electrical capacitance tomography[D]. Shenyang University of Technology, 2013(in Chinese).

[8] Yifan Wang. The research on sensor and reconstruction algorithm for direct three dimensional ECT[D]. Shenyang University of Technology, 2014(in Chinese).

[9] Zhendong Lin. Study on 3D ECT sensor structure parameters based on COMSOL[D]. Shenyang University of Technology, 2015(in Chinese).

[10] Ye Z, Wei H Y, Soleimani M. Resolution analysis using fully 3D electrical capacitive tomography[J]. Measurement, 2015, 61: 270-279.

[11] Xia Zhao, Xiaoyang Yu, Deyun Chen, et al. The capacitance measuring circuit for electrical capacitance tomography system[J]. Electrical measurement \& Instrumentation, 2002, 39(1): 2225(in Chinese).

[12] Yu Chen. Research on inverse problems solving and image reconstruction algorithm for electrical capacitance tomography system[D]. Harbin University of Science and Technology, 2010(in Chinese).

[13] Xin Xu. Optimization of sensor and research of reconstruction algorithm of direct 3D electrical capacitance tomography[D]. Shenyang University of Technology, 2014(in Chinese).

[14] Zepu Wang. Study on the image reconstruction for electrical capacitance tomography and the visualization measurement of the two phase flow in pneumatic conveying[D]. North China Electric Power University, 2013(in Chinese). 